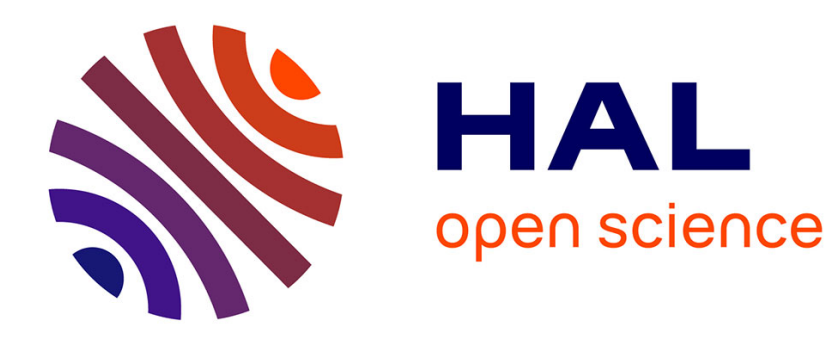

\title{
The weak-core of a game in normal form with a continuum of players
}

\author{
Youcef Askoura
}

\section{To cite this version:}

Youcef Askoura. The weak-core of a game in normal form with a continuum of players. Journal of Mathematical Economics, 2011, 47 (1), pp.43-47. 10.1016/j.jmateco.2010.11.003 . hal-01982380

\section{HAL Id: hal-01982380 \\ https://hal.science/hal-01982380}

Submitted on 15 Jan 2019

HAL is a multi-disciplinary open access archive for the deposit and dissemination of scientific research documents, whether they are published or not. The documents may come from teaching and research institutions in France or abroad, or from public or private research centers.
L'archive ouverte pluridisciplinaire HAL, est destinée au dépôt et à la diffusion de documents scientifiques de niveau recherche, publiés ou non, émanant des établissements d'enseignement et de recherche français ou étrangers, des laboratoires publics ou privés. 


\title{
The weak-core of a game in normal form with a continuum of players*
}

\author{
Y. Askoura
}

LEMMA, 4 rue Blaise Desgoffe, Paris 74006, France

y_askoura@yahoo.fr

\begin{abstract}
This paper deals with the weak-core of normal form games with a continuum set of players and without side payments. This concept is an approximation of the core introduced by Weber, Shapley and Shubik. The weak-core is slightly larger than Aumann's $\alpha$-core when adapted to large anonymous games. A non emptiness result is obtained based on the well known Scarf's non vacuity theorem for finite games.
\end{abstract}

keyword: Weak-Core; $\alpha$-Core; Game with a Continuum of Players; Large Anonymous Games; Normal Form Games JEL Classification codes : C02; C71.

\section{Introduction}

In this paper, we formulate the weak-core concept for strategic (normal form) games without side payments. The starting point of this work is the $\alpha$-core introduced by Aumann (1961) and investigated, for finite games, by Scarf (1967) and Kajii (1992). Precisely, we focus on an approximation of the $\alpha$-core introduced, as a quasi-core, by Shapley and Shubik (1966) for finite games and adapted to games with a continuum of players by Weber (1981). The studied games belong to the class of large anonymous games. The weakcore is slightly larger than the $\alpha$-core of Aumann (1961) and seems, in our context, more appropriate for mathematical treatment.

For finite (large) games in characteristic function form, the approximate solutions of the core are introduced in order to relax the convexity assumption generally assumed in studies of cores and competitive equilibria of exchange economies. It has been shown that the convexity (of preference sets) is not much important when the set of agents become large enough.

${ }^{*}$ This version includes some corrections to the original one, published in : Journal of Mathematical Economics 47(2011);43-47. 
In order to illustrate the intuitive idea of the different approximations of the core (known by the author) let us consider a game in characteristic function form $F=(N, \mathcal{V})$. Here $N$ is a finite set of players. In side payments case, $\mathcal{V}$ is a function which associate to each subset $S$ of $N$, a real number $\mathcal{V}(S)$. A coalition $S$ blocks an "allocation" $x \in \mathbb{R}^{N}$, if $\mathcal{V}(S)>\sum_{i \in S} x_{i}$. Then, the core of $F$ is the set of elements $x \in \mathbb{R}^{N}$ such that $\sum_{i \in N} x_{i}=\mathcal{V}(N)$ and $x$ cannot be blocked by any coalition $S \subset N$. Shapley and Shubik (1966) defined two approximate concepts : a weak $\varepsilon$-core and a strong $\varepsilon$-core. This was done by weakening the concept of blocking. For the weak $\varepsilon$ - core (resp. strong $\varepsilon-$ core), a coalition $S$ blocks $x \in \mathbb{R}^{N}$, if $\mathcal{V}(S)>\sum_{i \in S} x_{i}+|S| \varepsilon\left(\operatorname{resp} . \mathcal{V}(S)>\sum_{i \in S} x_{i}+\varepsilon\right)$. The notation $|S|$ stands for the cardinal of $S$. In non transferable utility case, the function $\mathcal{V}$ is a correspondence from the subsets of $N$ to that of $\mathbb{R}^{N}$. For this category of games, let us speak only about one approximation type (weak $\varepsilon$-core). Before defining it, an allocation $x$ is said to be blocked by a coalition $S$, if there exists $y \in \mathcal{V}(S)$ such that $y_{i}>x_{i}$ for every $i \in S$. Then, the core of $F$ is defined to be the elements of $\mathcal{V}(N)$ not blocked by any coalition. Let $\varepsilon>0$ be fixed. As defined in (Wooders, 2008), the blocking concept corresponding to the weak $\varepsilon$-core can be stated as follows : $x$ is blocked by a coalition $S \subset N$, if there exists $y \in \mathcal{V}(S)$ such that $y_{i}>x_{i}+\varepsilon$, for all $i \in S$.

The comparison between these approximations with the exact core is obvious. One can note that all these approximations contain the exact core and the latter corresponds to the particular case $\varepsilon=0$.

In transferable utility case, Shapley and Sbubik obtained interesting (non vacuity of approximate cores) results for exchange economies with sufficiently many participants. The concept of weak $\varepsilon$-core is successfully applied for (finite large) games induced from pergames (a configuration in which the payoff achievable by a coalition is a function of the number of its players and their characteristics or attributes) in (Wooders, 1983, 1994) and (Wooders and Zame, 1984). In case of a continuum set of players, Weber (1979) studied the concept of weak $\varepsilon$-core and proved its existence for balanced games in characteristic function form and without side payments.

A slightly different concept of weak $\varepsilon$-core is introduced by Kannai $(1970,1972)$ and applied for finite markets. The approach of Kannai consists in a representation of a finite market by a continuum one as defined in (Aumann, 1964). The non vacuity results are based on this representation. The approximation concept of Kannai is applied by Hildenbrand et al. (1973) to generalize the Shapley-Shubik results for large economies without side payments.

In a more recent paper, Wooders (2008) investigated the weak $\varepsilon$-core for (finite large) games (induced from pergames) without side payments. In (Wooders, 2008), the approximation is enlarged and deals, in addition, with the set of players. Roughly speaking, the approximation related to players was done by limiting the size of the possible blocking coalitions. More precisely, an element $x \in \mathcal{V}^{N}$ is in the weak $\varepsilon$-core if there exists $N_{0} \subset N$ such that $\left|N \backslash N_{0}\right| /|N|<\varepsilon$ and no coalition in $N_{0}$ can block $x$ (in the weak sens defined above). The approximation obtained by relaxing this limitation, is defined as the uniform 
$\varepsilon$-core by Wooders and its existence requires, as expected, additional conditions. Other studies about approximations of the core can be found in (Anderson, 1985; Starr, 1969).

It is obvious that these weak solutions can produce loss in stability, but as raised by the different authors, this can be offset, for example, by cooperation costs, which can be proportional to the number of cooperating players.

In this paper, we deal with Weber's approximation (Weber, 1981) as stated in Section 2 below. It is different from the above, its purpose is to "make easier" the passage from the finite to the continuum case rather than overcoming the nonexistence of the exact core caused by the non convexity assumption. Indeed, for finite games our weak core is exactly the $\alpha$-core of Aumann.

Following the idea of Scarf (1971), using a utility characteristic function game and another result of Scarf (1967), we show a non-emptiness result. However, we make some restrictions. We assume that the payoffs depend only on the distribution of the actions. Then, it is assumed that a payoff of an agent does not depend on its own action, but only on the resulting distribution of all (taken) actions.

For utility characteristic function games, there are very interesting results dealing with the continuum set of players case. We refer the reader, for instance, to Weber $(1981,1979)$, Ichiishi and Weber (1978), RosenmÂ,ller (1975) and Kaneko and Wooders (1996) (for particular games and by assuming that the admissible coalitions are finite). For more general frameworks concerning economies (exchange) and markets, we refer the reader to the famous works of Aumann (1964, 1966), Hildenbrand (1974, 1968), Mas-Colell (1975), Hart et al. (1974) and Khan (1981).

In a non cooperative setting, Schmeidler (1973) initiates the study of equilibria for games with a continuum of players. Mas-Colell (1984) developed this approach by defining the games by means of distributions on the set of agents' characteristics. The Mas-Colell equilibrium existence result has been generalized later by Khan (1989). By restraining the dependency of the agents' payoffs to the average of the actions, Rath (1992) obtained an existence result of a pure strategy equilibrium. We refer to Khan et al. (1997) for a complete review on Nash equilibria for games with a continuum set of agents.

\section{Main result}

By a normal form (strategic) game with a continuum of players, we mean a triple

$$
G=\left((T, \lambda), \mathcal{U}_{A}, A\right)
$$

$T=[0,1]$ is the set of players endowed with its usual topology. $\lambda$ stands for the Lebesgue measure on $T$. $A$ is the set of actions. Assume that $A$ is a compact metric space. Let us denote by $\mathcal{M}_{+}^{1}(A)$ the set of Radon probability measures on $A$ endowed with the weak (star) topology. Since $A$ is a Suslin space, all Borel measures on $A$ are Radon (Bogachev, 2007). Then, $\mathcal{M}_{+}^{1}(A)$ is the set of all Borel probability measures on $A$. In the sequel, other sets of positive Radon measures on $A$ are used. They are always considered as subspaces 
of the space $\mathcal{M}_{+}(A)$ of all Radon positive measures on $A$ endowed with the weak topology. Note that the convexity of $A$ is not needed in this paper. We use, instead, the convexity of $\mathcal{M}_{+}^{1}(A)$ which is more advantageous.

The players' utility functions are described by a function $u: T \times \mathcal{M}_{+}^{1}(A) \rightarrow \mathbb{R}$. We assume that $u$ is continuous with respect to the resulting product topology. To each player $t$ corresponds its utility function $u(t,):. \mathcal{M}_{+}^{1}(A) \rightarrow \mathbb{R}$. In the sequel, this function is denoted by $u(t)$. When each player $t$ chooses its strategy $f(t)$, we obtain a function $f$, $f(t) \in A, t \in T$. Assuming that this function is measurable, each player $t$ receives the gain $u(t)\left(\lambda f^{-1}\right)$. Here, $\lambda f^{-1}$ stands for the image measure of $\lambda$ under the function $f$. Then, $u(t)$ depends only on the distribution $\lambda f^{-1}$. The set of utility functions $u(t), t \in T$, is denoted by $\mathcal{U}_{A}$ above and is endowed with the sup-norm topology. Thus, the continuity of $u$ yields that of the function $t \mapsto u(t)$ from $T$ to $\mathcal{U}_{A}$.

Let $\mathfrak{B}(T)$ be the set of all Borel subsets of $T$. The set of permissible coalitions is the set of Borel subsets of $T$ of strictly positive $\lambda$-measure, denote it by $\mathfrak{B}_{+}(T)$. For a Borel subset $X$ of $T$, we denote by $\mathfrak{B}(X, A)$ the set of Borel functions defined from $X$ into $A$. If $X_{1}$ and $X_{2}$ are two disjoint Borel subsets of $T, h_{X_{1}} \in \mathfrak{B}\left(X_{1}, A\right)$ and $h_{X_{2}} \in \mathfrak{B}\left(X_{2}, A\right)$, then $h=\left(h_{X_{1}}, h_{X_{2}}\right)$ stands for the Borel function defined as $h_{X_{1}}$ on $X_{1}$ and $h_{X_{2}}$ on $X_{2}$. Clearly, $h \in \mathfrak{B}\left(X_{1} \cup X_{2}, A\right)$. The complement of a subset $X$ of a given space $Y$ is denoted by $Y \backslash X$ or simply $\complement X$ (if no confusion may occur). For instance, all complements of subsets of $T$ stand for complements to $T$. In the whole of this paper, the measurability is taken with respect to the Borel $\sigma$-algebra. The closure of a subset $X$ in a topological space is denoted by $\bar{X}$. If $c$ is a real number and $X$ a subset of a linear space, then $c . X$ stands for the set $\{c x: x \in X\}$.

Definition 1. We say that a coalition $E \in \mathfrak{B}_{+}(T)$ blocks the strategy $f \in \mathfrak{B}(T, A)$ (or the distribution $\left.p=\lambda f^{-1}\right)$, if there is $\varepsilon>0$ and a Borel function $h_{E} \in \mathfrak{B}(E, A)$, such that for every $h_{\complement E} \in \mathfrak{B}(\complement E, A)$, letting $h=\left(h_{E}, h_{\complement E}\right)$, we have :

$$
u(t)\left(\lambda h^{-1}\right)>u(t)\left(\lambda f^{-1}\right)+\varepsilon \text { a.e. on } E
$$

The weak-core of the game $G$ is the set of distributions that are not blocked by any coalition $E \in \mathfrak{B}_{+}(T)$.

Following the representation result of Skorokhod (1956) (see also (Blackwell and Dubins, 1983; Bogachev, 2007)), for each element $p \in \mathcal{M}_{+}^{1}(A)$, there corresponds a function $f \in$ $\mathfrak{B}(T, A)$, such that $p=\lambda f^{-1}$. Then the weak-core can be defined either by means of measures $p \in \mathcal{M}_{+}^{1}(A)$ or by functions in $\mathfrak{B}(T, A)$. Note that in this last case, there may be many functions corresponding to a same probability $p \in \mathcal{M}_{+}^{1}(A)$. Our definition is more precise with measures. When speaking about the weak-core in terms of functions (strategies), we mean a class of functions inducing the same probability. Furthermore, considering the blocking concept from the previous definition, any two coalitions having a null set as a symmetric difference can be confused.

The weak-core is slightly larger than the $\alpha$-core of Aumann (1961). More precisely, if we remove $\varepsilon$ in the previous definition, we obtain naturally the $\alpha$-core in case of a continuum 
set of players. Moreover, one can remark easily, as mentioned in (Weber, 1981), that for finite games (finite set of players) these two concepts coincide. Our proof of existence works only for the weak-core. In our context, the proof of Lemma 1 below fails if we remove $\varepsilon$ in Definition 1.

Theorem 1. Under the hypotheses described above and the concavity of the functions $p \mapsto u(t)(p)$ for all $t \in T$, the weak-core of $G$ is non empty.

Associate to each $E \in \mathfrak{B}_{+}(T)$, the set

$$
\mathcal{H}(E)=\left\{p \in \mathcal{M}_{+}^{1}(A): p \text { is not blocked by } E\right\}
$$

In order to prove that the weak-core of $G$ is non empty, we need to prove that the intersection $\bigcap_{E \in \mathfrak{B}_{+}(T)} \mathcal{H}(E)$ is non empty. Since $\mathcal{M}_{+}^{1}(A)$ is compact for the weak topology, it suffices to prove that the sets $\mathcal{H}(E)$ are closed and every intersection of a finite subfamily $\mathcal{H}\left(E_{i}\right), i \in I$, is non empty. This is the objective of the following two lemmas :

Lemma 1. For every $E \in \mathfrak{B}_{+}(T), \mathcal{H}(E)$ is closed in $\mathcal{M}_{+}^{1}(A)$.

Proof. Let $E \in \mathfrak{B}_{+}(T)$ and $p \in \mathcal{C H}(E)$. Then, there exist $\varepsilon>0$ and $h_{E} \in \mathfrak{B}(E, A)$ such that,

$$
\begin{aligned}
& \text { for all } h_{\complement E} \in \mathfrak{B}(\complement E, A), \text { for } h=\left(h_{E}, h_{\complement E}\right), \\
& u(t)\left(\lambda h^{-1}\right)>u(t)(p)+\varepsilon, \text { for a.e. } t \in E
\end{aligned}
$$

By the Skorokhod representation theorem (Skorokhod, 1956; Blackwell and Dubins, 1983; Bogachev, 2007), the set of measures on $A$ obtained as images of $\lambda$ by the Borel functions $h_{\complement E} \in \mathfrak{B}(\complement E, A)$ is

$$
\lambda(\complement E) \cdot \mathcal{M}_{+}^{1}(A)
$$

Indeed, if $\lambda(E)=\lambda(T)$, then the unique possible measure obtained by the functions $h_{\complement E} \in \mathfrak{B}(\complement E, A)$ is the null measure. Otherwise, $\lambda(\complement E)>0$. From Proposition 9.1.11 in Bogachev (2007), there is a Borel function $\varphi: \complement E \rightarrow T$, such that $\lambda_{\mid \subset E} \varphi^{-1}=\lambda(\complement E) . \lambda$. Then by Skorokhod's representation theorem, the set of measures $\lambda_{\mid C E}(f \circ \varphi)^{-1}$ obtained when $f$ ranges $\mathfrak{B}(T, A)$ is $\lambda(\complement E) . \mathcal{M}_{+}^{1}(A)$. Thus, the set of measures, $\lambda h^{-1}$, appearing in Formula (2) is exactly the set

$$
R\left(E, h_{E}\right)=\mu_{E}+\lambda(\complement E) \cdot \mathcal{M}_{+}^{1}(A)
$$

where $\mu_{E}=\lambda h_{E}^{-1}$. Clearly $R\left(E, h_{E}\right)$ is a compact set.

In terms of measures, the formula (2) is equivalent to the following :

$$
\forall q \in R\left(E, h_{E}\right), u(t)(q)>u(t)(p)+\varepsilon, \text { for a.e. } t \in E
$$

For every $q \in R\left(E, h_{E}\right)$, let

$$
E_{q}=\{t \in E: u(t)(q)>u(t)(p)+\varepsilon\}
$$


Note that, for all $q \in R\left(E, h_{E}\right), E_{q}$ is measurable as intersection of an open set and $E$ and $\lambda\left(E_{q}\right)=\lambda(E)$.

Using the continuity of $u$, we can state that for all $t \in \overline{E_{q}}, u(t)(q) \geq u(t)(p)+\varepsilon$. Choose $\left.\varepsilon_{1} \in\right] 0, \varepsilon\left[\right.$. Using the continuity of $u$ again, we can find, for every $q \in R\left(E, h_{E}\right)$, two neighborhoods $\theta_{q}, \theta_{p}(q)$ of $q$ and $p$ respectively such that,

$$
\forall t \in \overline{E_{q}}, \forall q^{\prime} \in \theta_{q}, \forall p^{\prime} \in \theta_{p}(q), u(t)\left(q^{\prime}\right)>u(t)\left(p^{\prime}\right)+\varepsilon_{1}
$$

Let us consider all the sets $E_{q}, \theta_{q}, \theta_{p}(q)$ constructed as above. Since $R\left(E, h_{E}\right)$ is compact, there exists a finite subfamily $\left\{\theta_{q_{i}}, i \in I\right\}$ of the family $\left\{\theta_{q}, q \in R\left(E, h_{E}\right)\right\}$ covering $R\left(E, h_{E}\right)$. Let $E^{\prime}=\bigcap_{i \in I} \overline{E_{q_{i}}} \cap E, \theta_{R\left(E, h_{E}\right)}=\cup_{i \in I} \theta_{q_{i}}$ and $\theta_{p}=\bigcap_{i \in I} \theta_{p}\left(q_{i}\right)$.

We obtained

$$
\forall p^{\prime} \in \theta_{p}, \forall t \in E^{\prime}, \forall q \in \theta_{R\left(E, h_{E}\right)}, u(t)(q)>u(t)\left(p^{\prime}\right)+\varepsilon_{1}
$$

Moreover, it is obvious that $\lambda\left(E^{\prime}\right)=\lambda(E)$. Hence, we can replace in the previous formula $t \in E^{\prime}$ by the expression "for a.e. $t \in E$ " together with other simplifications, we obtain : for all $p^{\prime} \in \theta_{p}$,

$$
\forall q \in R\left(E, h_{E}\right), u(t)(q)>u(t)\left(p^{\prime}\right)+\varepsilon_{1} \text { for a.e. } t \in E
$$

It results that $E$ blocks all the measures in $\theta_{p}$. This proves that the complement of $\mathcal{H}(E)$ is open and ends the proof.

Lemma 2. Let $I$ be a finite set of indices and for each $i \in I$, a coalition $E_{i} \in \mathfrak{B}_{+}(T)$. Consider the sets $\mathcal{H}\left(E_{i}\right), i \in I$, defined in (1). Assume that the functions $p \mapsto u(t)(p)$, $t \in T$, are concave. Then, $\bigcap_{i \in I} \mathcal{H}\left(E_{i}\right) \neq \emptyset$.

Proof. Let $K_{j}, j \in J$, be a finite family of piecewise disjoint measurable subsets of $T$ of strictly positive measure, such that every $E_{i}$ is an union (up to a $\lambda$-null set) of some sets $K_{j}$. For a subset $B \subset J$, we denote $K_{B}=\bigcup_{j \in B} K_{j}$. Furthermore, we can assume that $\bigcup_{j \in J} K_{j}=T$ up to a $\lambda$-null set. Indeed, if it is not the case, one can add the set $\mathcal{H}(T)$ to the family $\mathcal{H}\left(E_{i}\right), i \in I$.

For each $j \in J$, denote for every $\rho \in \mathcal{M}_{+}^{1}(A)$,

$$
g_{j}(\rho)=\int_{K_{j}} u(t)(\rho) d \lambda(t)
$$

Note that the functions $g_{j}$ are concave on $\mathcal{M}_{+}^{1}(A)$. This follows from the concavity of the functions $u(t)$. Also, the functions $g_{j}$ are continuous. This fact can be verified by applying, for example, Lebesgue's dominated convergence theorem.

In the sequel, we follow the idea of Scarf (1971) using a utility characteristic function game and another result of Scarf (1967). 
Associate the utility characteristic function game $G_{1}=(J, V)$, where, for every $B \subseteq J$, $V(B)$ is the subset of $\mathbb{R}^{J}$ defined by :

$$
v \in V(B) \Leftrightarrow \mid \begin{aligned}
& \exists h_{K_{B}} \in \mathfrak{B}\left(K_{B}, A\right), \forall h_{\complement K_{B}} \in \mathfrak{B}\left(\complement K_{B}, A\right), \text { for } h=\left(h_{K_{B}}, h_{\complement K_{B}}\right), \\
& g_{j}\left(\lambda h^{-1}\right) \geq v_{j}, \forall j \in B
\end{aligned}
$$

A vector $v \in \mathbb{R}^{J}$ is in the core of the game $G_{1}$ if $v \in V(J)$ and $v$ is not in the interior of $V(B)$ for every $B \subset J$. It is easy to verify that $G_{1}$ has the following properties :

1) For every $B \subset J, V(B)$ is closed and nonempty,

2) For every $B \subset J$, if $v \in V(B)$ and $v^{\prime} \leq v$ then $v^{\prime} \in V(B)$,

3) $V(J)$ is bounded from above.

Let us verify the closure of the sets $V(B), B \subset J$. Their non emptiness and the other items are straightforward. Let $B \subset J$ be fixed. Similarly to Lemma 1, by applying the Skorokhod representation theorem, $v \in V(B)$ if there exists $\mu_{B} \in \lambda\left(K_{B}\right)$. $\mathcal{M}_{+}^{1}(A)$ such that for all $\rho \in \lambda\left(\complement K_{B}\right) . \mathcal{M}_{+}^{1}(A)$, for all $j \in B, g_{j}\left(\mu_{B}+\rho\right) \geq v_{j}$. Thus, from the continuity of the functions $g_{j}$ and the compactness of the sets $\lambda\left(K_{B}\right) \cdot \mathcal{M}_{+}^{1}(A)$ and $\lambda\left(\complement K_{B}\right) \cdot \mathcal{M}_{+}^{1}(A)$, we can write $V(B)$ as :

$$
V(B)=\left\{v \in \mathbb{R}^{J}: \max _{\mu_{B} \in \lambda\left(K_{B}\right) \cdot \mathcal{M}_{+}^{1}(A)} \min _{\rho \in \lambda\left(\complement K_{B}\right) \cdot \mathcal{M}_{+}^{1}(A)} \min _{j \in B}\left(g_{j}\left(\mu_{B}+\rho\right)-v_{j}\right) \geq 0\right\}
$$

It follows that the sets $V(B), B \subset J$, are closed. Note further that if $v \in \mathbb{R}^{J}$ is not in the interior of $V(B)$, for a given $B \subset J$, then,

$$
\begin{aligned}
& \forall h_{K_{B}} \in \mathfrak{B}\left(K_{B}, A\right), \exists h_{\complement K_{B}} \in \mathfrak{B}\left(\complement K_{B}, A\right), \text { for } h=\left(h_{K_{B}}, h_{\complement K_{B}}\right), \\
& \exists j \in B \text {, such that } g_{j}\left(\lambda h^{-1}\right) \leq v_{j}
\end{aligned}
$$

The game $G_{1}$ is said to be balanced (Scarf, 1971, 1967) if

$$
\bigcap_{B \in \mathfrak{C}} V(B) \subset V(J)
$$

for every balanced collection of coalitions $\mathfrak{C}$. Note that a collection of coalitions $\mathfrak{C}$ is said to be balanced provided there exist non negative weights $\delta_{B}, B \in \mathfrak{C}$, such that

$$
\sum_{B \in \mathfrak{C}, B \ni j} \delta_{B}=1, \forall j \in J
$$

Remark that the definition of balancedness of a game does not change if we consider only balanced coalitions with strictly positives weights. 
Following Scarf (1967), the core of $G_{1}$ is nonempty under the hypothesis of balancedness and the conditions 1)-3) above. Then, for the non vacuity of the core of $G_{1}$, it remains only to prove its balancedness. Let, for this aim, $\mathfrak{C}$ be a balanced family of coalitions of $G_{1}$, with strictly positive balancing coefficients $\delta_{S}, S \in \mathfrak{C}$, and $v \in \underset{S \in \mathfrak{C}}{\cap} V(S)$. Then, for every $S \in \mathfrak{C}$, there is a Borel function $h_{K_{S}} \in \mathfrak{B}\left(K_{S}, A\right)$ such that, for every $j \in S$, $g_{j}\left(\lambda\left(h_{K_{S}}, h_{\complement K_{S}}\right)^{-1}\right) \geq v_{j}$, for all $h_{\complement K_{S}} \in \mathfrak{B}\left(\complement K_{S}, A\right)$. Put $\mu_{S}=\lambda h_{K_{S}}^{-1}$. Observe that $\mu_{S}$ is a Radon measure on $A$. By the Skorokhod representation theorem, for every positive measure $\rho$, such that $\mu_{S}+\rho \in \mathcal{M}_{+}^{1}(A)$, there is a measurable function $h_{\complement K_{S}} \in \mathfrak{B}\left(\complement K_{S}, A\right)$ such that $\lambda h_{\complement K_{S}}^{-1}=\rho$ (see also the proof of Lemma 1). Letting $h=\left(h_{K_{S}}, h_{\complement K_{S}}\right)$, we obtain $\lambda h^{-1}=\mu_{S}+\rho$.

Thus,

$$
g_{j}\left(\mu_{S}+\rho\right) \geq v_{j}, \forall j \in S
$$

Put

$$
\mu=\sum_{S \in \mathfrak{C}} \delta_{S} \mu_{S}
$$

In the sequel, we try to express, for a given $j \in J, \mu$ as a convex combination of probabilities with the form $\mu_{S}+\rho, S \ni j$. Before this, we have

$$
\mu(A)=\sum_{S \in \mathfrak{C}} \delta_{S} \mu_{S}(A)=\sum_{S \in \mathfrak{C}} \delta_{S} \lambda\left[h_{K_{S}}^{-1}(A)\right]=\sum_{S \in \mathfrak{C}} \delta_{S} \lambda\left(K_{S}\right)=\sum_{S \in \mathfrak{C}} \delta_{S} \lambda\left(\bigcup_{j \in S} K_{j}\right)
$$

This sum can be rewritten as :

$$
\mu(A)=\sum_{S \in \mathfrak{C}} \delta_{S} \lambda\left(\bigcup_{j \in S} K_{j}\right)=\sum_{S \in \mathfrak{C}} \delta_{S} \sum_{j \in S} \lambda\left(K_{j}\right)=\sum_{j \in J} \sum_{S \ni j} \delta_{S} \lambda\left(K_{j}\right)
$$

From the balancedness of $\mathfrak{C}$, the last equality reduces to $\mu(A)=\sum_{j \in J} \lambda\left(K_{j}\right)$. Since $T=\bigcup_{j \in J} K_{j}$ up to a null set, $\lambda(T)=\sum_{j \in J} \lambda\left(K_{j}\right)$. Hence, $\mu(A)=1$. Then $\mu$ is a Radon probability on $A$. Fix an arbitrary index $j \in J$. We have

$$
\mu=\sum_{S \in \mathfrak{C}, S \ni j} \delta_{S} \mu_{S}+\sum_{S \in \mathfrak{C}, j \notin S} \delta_{S} \mu_{S}
$$

Let us denote

$$
\mu_{-j}=\sum_{S \in \mathfrak{C}, j \notin S} \delta_{S} \mu_{S}
$$

Since $\sum_{S \in \mathfrak{C}, S \ni j} \delta_{S}=1$, we can write

$$
\mu=\left(\sum_{S \in \mathfrak{C}, S \ni j} \delta_{S} \mu_{S}\right)+\mu_{-j}=\sum_{S \in \mathfrak{C}, S \ni j} \delta_{S}\left(\mu_{S}+\mu_{-j}\right)
$$


If, for each $S \ni j, \mu_{S}+\mu_{-j}$ is a probability, from the foregoing, we can use the concavity of the function $g_{j}$ to conclude that $g_{j}(\mu) \geq v_{j}$ and then the balancedness of the game. But, this is not necessarily true. In order to overcome this difficulty, let us transform another time the measure $\mu$. Denote

$$
\begin{aligned}
& \mathfrak{C}_{j}^{+}=\left\{S \in \mathfrak{C}: S \ni j \text { and } \mu_{S}(A)+\mu_{-j}(A)>1\right\} \\
& \mathfrak{C}_{j}^{-}=\left\{S \in \mathfrak{C}: S \ni j \text { and } \mu_{S}(A)+\mu_{-j}(A)<1\right\}
\end{aligned}
$$

and

$$
\mathfrak{C}_{j}^{00}=\left\{S \in \mathfrak{C}: S \ni j \text { and } \mu_{S}(A)+\mu_{-j}(A)=1\right\}
$$

Since $\mu(A)=1$, if $\mathfrak{C}_{j}^{+}=\emptyset$, necessarily $\mathfrak{C}_{j}^{-}=\emptyset$. Then, all the measures $\mu_{S}+\mu_{-j}$ are probability measures. Assume it is not the case. Since $\mu_{S}(A) \leq 1$, for all $S \in \mathfrak{C}$, necessarily $\mu_{-j}(A)>0$. Let us prove also that in this circumstance $\mu$ can be written as a convex combination of the needed probability measures.

For all $S \in \mathfrak{C}_{j}^{+}$, let $\alpha_{S} \in[0,1]$, such that $\mu_{S}(A)+\mu_{-j}(A)-\alpha_{S} \mu_{-j}(A)=1$. The existence of $\alpha_{S}$ follows from the fact that $S \in \mathfrak{C}_{j}^{+}, \mu_{S}(A) \leq 1$ and $\mu_{-j}(A)>0$.

For all $S \in \mathfrak{C}_{j}^{-}$, let $\beta_{S}>0$, such that $\mu_{S}(A)+\mu_{-j}(A)+\beta_{S} \mu_{-j}(A)=1$.

Then,

$$
\begin{aligned}
1= & \sum_{S \in \mathfrak{C}_{j}^{+}} \delta_{S}\left[\mu_{S}(A)+\mu_{-j}(A)-\alpha_{S} \mu_{-j}(A)\right] \\
& +\sum_{S \in \mathfrak{C}_{j}^{-}} \delta_{S}\left[\mu_{S}(A)+\mu_{-j}(A)+\beta_{S} \mu_{-j}(A)\right] \\
& +\sum_{S \in \mathfrak{C}_{j}^{0}} \delta_{S}\left[\mu_{S}(A)+\mu_{-j}(A)\right] \\
= & \sum_{S \ni j} \delta_{S}\left(\mu_{S}(A)+\mu_{-j}(A)\right)+\mu_{-j}(A)\left[-\sum_{S \in \mathfrak{C}_{j}^{+}} \delta_{S} \alpha_{S}+\sum_{S \in \mathfrak{C}_{j}^{-}} \delta_{S} \beta_{S}\right]
\end{aligned}
$$

Since $\mu(A)=1$ and $\mu_{-} j(A)>0$, we conclude from (4) that

$$
-\sum_{S \in \mathfrak{C}_{j}^{+}} \delta_{S} \alpha_{S}+\sum_{S \in \mathfrak{C}_{j}^{-}} \delta_{S} \beta_{S}=0
$$

This equation establishes that we can write $\mu$ as :

$$
\mu=\sum_{S \in \mathfrak{C}_{j}^{+}} \delta_{S}\left[\mu_{S}+\mu_{-j}-\alpha_{S} \mu_{-j}\right]+\sum_{S \in \mathfrak{C}_{j}^{-}} \delta_{S}\left[\mu_{S}+\mu_{-j}+\beta_{S} \mu_{-j}\right]+\sum_{S \in \mathfrak{C}_{j}^{0}} \delta_{S}\left[\mu_{S}+\mu_{-j}\right]
$$

That is, $\mu$ is a convex combination of probability measures and each one, associated to $S \ni j$, is in the form

$$
\mu_{S}+\rho
$$

where $\rho$ is a positive measure. Then $g_{j}\left(\mu_{S}+\rho\right) \geq v_{j}$. From the concavity of $g_{j}$, we conclude that $g_{j}(\mu) \geq v_{j}$. Since $j$ is arbitrarily chosen in $J$, we conclude that $g_{j^{\prime}}(\mu) \geq v_{j^{\prime}}$, for all $j^{\prime} \in J$. To complete the proof of the balancedness of $G_{1}$, it suffices to apply again the 
Skorokhod representation theorem to establish that there is a function $h \in \mathfrak{B}(T, A)$, such that $\mu=\lambda h^{-1}$. Thus, we have proved that the core of $G_{1}$ is nonempty.

To end the proof of the lemma, remark that an element of the core of $G_{1}$ provides necessarily an element of the intersection $\bigcap_{i \in I} \mathcal{H}\left(E_{i}\right)$. Indeed, let $v$ be an element of the core of $G_{1}$ and $f \in \mathfrak{B}(T, A)$ such that $g_{j}\left(\lambda f^{-1}\right) \geq v_{j}$, for all $j \in J$. Put again $\mu=\lambda f^{-1} \in \mathcal{M}_{+}^{1}(A)$. Fix and arbitrary index $i \in I . \quad E_{i}$ is a finite union (up to a $\lambda$-null set) of some sets $K_{j}, j \in J_{i}$. Since $v$ belongs to the core of $G_{1}, v$ cannot be in the interior of $V\left(J_{i}\right)$. Hence, for every $h_{E_{i}} \in \mathfrak{B}\left(E_{i}, A\right)$, there exist $h_{\complement E_{i}} \in \mathfrak{B}\left(\complement E_{i}, A\right)$ and an index $j \in J_{i}$ such that for $h=\left(h_{E_{i}}, h_{\complement E_{i}}\right)$,

$$
\int_{K_{j}} u(t)\left(\lambda h^{-1}\right) d \lambda(t) \leq v_{j} \leq \int_{K_{j}} u(t)(\mu) d \lambda(t)
$$

Thus, for every $h_{E_{i}} \in \mathfrak{B}\left(E_{i}, A\right)$, there exists $h_{\complement E_{i}} \in \mathfrak{B}\left(\complement E_{i}, A\right)$ and a measurable subset $D_{i}$ of $E_{i}$ of strictly positive measure, such that for $h=\left(h_{E_{i}}, h_{\complement E_{i}}\right)$,

$$
u(t)\left(\lambda h^{-1}\right) \leq u(t)(\mu), \forall t \in D_{i}
$$

This proves that $\mu \in \mathcal{H}\left(E_{i}\right)$, which completes the proof.

\section{Acknowledgments}

The author is very grateful for precise and various comments of an anonymous referee and an associate editor. Thanks to them, the content of this paper has been significantly improved.

\section{References}

Anderson R M. Strong core theorems with nonconvex preferences. Econometrica 1985; 53; 1283-1294.

Aumann R J. The core of a cooperative game without side payments. Transactions of the American Mathematical Society 1961; 98; 539-552.

Aumann R J. Markets with a continuum of traders. Econometrica 1964; 32; 39-50.

Aumann R J. Existence of competitive equilibria in markets with a continuum of traders. Econometrica $1966 ; 34 ; 1-17$.

Blackwell D and Dubins L E. An extension of Skorohod's almost sure representation theorem. Proceedings of the American Mathematical Society 1983; 89; 691-692.

Bogachev V I. Measure theory (2 volumes). Springer-Verlag, Berlin, 2007. 
Hart S, Hildenbrand W and Kohlberg E. On equilibrium allocations as distributions on the commodity space. Journal of Mathematical Economics 1974; 1; 159-166.

Hildenbrand $\mathrm{W}$. The core of an economy with a measure space of economic agents. The Review of Economic Studies 1968; 35; 443-452.

Hildenbrand W. Core and equilibria of a large economy. Princeton University Press, Princeton, N. J., 1974.

Hildenbrand W, Schmeidler D and Zamir S. Existence of approximate equilibria and cores. Econometrica $1973 ; 41 ; 1159-1166$.

Ichiishi $\mathrm{T}$ and Weber S. Some theorems on the core of a non-sidepayment game with a measure space of players. International Journal of Game Theory 1978; 7; 95-112.

Kajii A. A generalization of Scarf's theorem : an $\alpha$-core existence theorem without transitivity or completeness. Journal of Economic Theory 1992; 56; 194-205.

Kaneko M and Wooders M H. The nonemptiness of the f-core of a game without side payments. International Journal of Game Theory 1996; 25; 245-258.

Kannai Y. Continuity properties of the core of a market. Econometrica 1970; 38; 791-815.

Kannai Y. Continuity properties of the core of a market : a correction. Econometrica 1972; 40; 955-958.

Khan M A. On the cores of economies with indivisible commodities and a continuum of traders. Journal of Economic Theory 1981; 24; 218-225.

Khan M A. On a Cournot-Nash equilibrium distributions for games with a nonmetrizable action space and upper semicontinuous payoffs. Transactions of the American Mathematical Society 1989; 315; 127-146.

Khan M A, Rath K P and Sun Y. On the existence of pure strategy equilibria in games with a continuum of players. Journal of Economic Theory 1997; 76; 13-46.

Mas-Colell A. A model of equilibrium with differentiated commodities. Journal of Economic Theory $1975 ; 2 ; 263-295$.

Mas-Colell A. On a theorem of Schmeidler. Journal of Mathematical Economics 1984; 13; 201-206.

Rath K P. A direct proof of the existence of pure strategy equilibria in games with a continuum of players. Economic Theory 1992; 2; 427-433.

RosenmÂ,ller J. Large games without side payments. Operations Research Verfahren, Band XX, pp. 107-128, Hain, Meisenheim am Glan, 1975. 
Scarf H E. The core of an n-person game. Econometrica 1967; 35; 50-69.

Scarf H E. On the existence of a cooperative solution for a general class of N-person games. Journal of Economic Theory 1971; 3; 169-181.

Schmeidler D. Equilibrium points of nonatomic games. Journal of Statistical Physics 1973; 7; 295-300.

Shapley L. On balanced sets and cores. The RAND Corporation, Santa Monica, California, Memorandum RM-4601-PR (June 1965).

Shapley L S and Shubik M. Quasi-core in a monetary economy with nonconvex preferences. Econometrica 1966; 34; 805-828.

Skorokhod A V. Limit theorems for stochastic processes. Theory of Probability and its Applications 1956; 1; 261-290.

Starr R M. Quasi-equilibria in markets with non-convex preferences. Econometrica 1969; 37; $25-38$.

Weber S. Some results on the weak core of a non-side-payment game with infinitely many players. Journal of Mathematical Economics 1981; 8; 101-111.

Weber S. On $\epsilon$-core of balanced games. International Journal of Game Theory 1979; 8; 241-250.

Wooders M H. The epsilon core of a large replica game. Journal of Mathematical Economics $1983 ; 11 ; 277-300$.

Wooders M H and Zame W R. Approximate cores of large games. Econometrica 1984; 52; $1327-1350$.

Wooders M H. Large games and economies with effective small groups. In: Mertens, J.F., Sorin, S. (Eds.), Game-Theoretic Methods in General Equilibrium Analysis. Kluwer Academic Publishers, Dordrecht, Boston, London, pp. 145-206; 1994.

Wooders M H. Small group effectiveness, per capita boundedness and nonemptiness of approximate cores. Journal of Mathematical Economics 2008; 44; 888-906. 